\title{
Poincaré recurrences and Ulam method for the Chirikov standard map
}

\author{
Klaus M. Frahm and Dima L. Shepelyansky \\ 1 Laboratoire de Physique Théorique du CNRS, IRSAMC, Université de Toulouse, UPS, 31062 Toulouse, France \\ 2 http://www.quantware.ups-tlse.fr
}

February 12, 2013

\begin{abstract}
We study numerically the statistics of Poincaré recurrences for the Chirikov standard map and the separatrix map at parameters with a critical golden invariant curve. The properties of recurrences are analyzed with the help of a generalized Ulam method. This method allows to construct the corresponding Ulam matrix whose spectrum and eigenstates are analyzed by the powerful Arnoldi method. We also develop a new survival Monte Carlo method which allows us to study recurrences on times changing by ten orders of magnitude. We show that the recurrences at long times are determined by trajectory sticking in a vicinity of the critical golden curve and secondary resonance structures. The values of Poincaré exponents of recurrences are determined for the two maps studied. We also discuss the localization properties of eigenstates of the Ulam matrix and their relation with the Poincaré recurrences.
\end{abstract}

PACS. 05.45.Ac Low-dimensional chaos - 05.45.Pq Numerical simulations of chaotic systems - 05.45.Fb Random walks and Levy flights

\section{Introduction}

The interest to understanding of transition from dynamical to statistical description of motion had started from the dispute between Loschmidt and Boltzmann, which is now known as the Loschmidt paradox [1,2]. The twodimensional (2D) symplectic maps represent an excellent laboratory for investigation of how statistical laws appear in dynamical, fully deterministic systems. Their properties have been studied in great detail during last decades both on mathematical (see e.g. 3, 4 and Refs. therein) and physical (see e.g. [5, 6, 7, and Refs. therein) levels of rigor. The case of completely chaotic behavior, appearing e.g. in Anosov systems, is now well understood 3, 4 but a generic case of maps with divided phase space, where islands of stability are surrounded by chaotic components, still preserves its puzzles. A typical example of such a map is the Chirikov standard map [5, 6] which often gives a local description of dynamical chaos in other dynamical maps and describes a variety of physical systems (see e.g. [8]). This map has the form:

$$
\bar{y}=y+\frac{K}{2 \pi} \sin (2 \pi x), \quad \bar{x}=x+\bar{y}(\bmod 1) .
$$

Here $x, y$ are canonical conjugated variables of generalized phase and action, bars mark the variables after one map iteration and we consider the dynamics to be periodic on a torus so that $0 \leq x \leq 1,0 \leq y \leq 1$. The dynamics is characterized by one dimensionless chaos parameter $K$.
For small values of $K$ the phase space is covered by invariant Kolmogorov-Arnold-Moser (KAM) curves which restrict dynamics in the action variable $y$. For $K>K_{g}$ the last invariant golden curve with the rotation number $r=r_{g}=\left\langle\left(x_{t}-x_{0}\right) / t\right\rangle=(\sqrt{5}-1) / 2$ is destroyed [9, 10] and it is believed that for $K>K_{g}$ the dynamics in $y$ becomes unbounded [11,12]. A renormalization technique developed by Greene and MacKay [9, 10, allowed to determine $K_{g}=0.971635406$ with enormous precision. The properties of the critical golden curve on small scales are universal for all critical curves with the golden tail of the continuous fraction expansion of $r$ for all smooth 2D symplectic maps [10]. Here and below the time $t$ is measured in number of map iterations (due to symmetry there is also a symmetric critical curve at $r=1-r_{g}$ at $K_{g}$ ). For $K>K_{g}$ the golden KAM curve is replaced by a cantori [13] which can significantly affect the diffusive transport through the chaotic part of the phase space [14,15]. At any $K$ there are some chaotic regions in the phase space bounded by internal or isolating (at $K<K_{g}$ ) invariant curves.

The dynamics inside a chaotic component of the phase space $(x, y)$ is characterized by correlation functions whose decay ensures a transition from dynamical to statistical description. The decay of correlations is related to the probability to stay in a given region of phase space since for a trajectory remaining in a small region the dynamical variables are strongly correlated. This probability in its own turn is related to the statistics of Poincaré recurrences. Indeed, according to the Poincaré recurrence 
theorem [16] a dynamical trajectory of a Hamiltonian system with bounded phase space always returns, after a certain time, to a close vicinity of an initial state. However, the statistics of these recurrences depends on dynamical properties of the system. For a fully chaotic phase space a probability to stay in a certain part of a phase space decays exponentially with time being similar to a random coin flipping 3, 4. However, in dynamical maps with divided phase space, like the Chirikov standard map, the decay of probability of Poincaré recurrences $P(t)$ is characterized by a power law decay $P(t) \propto 1 / t^{\beta}$ has $\beta \approx 1.5$ whose properties still remain poorly understood.

One of the first studies of Poincaré recurrences in dynamical Hamiltonian systems with two degrees of freedom was done in 17. where an algebraic decay with an exponent $\beta=1 / 2$ was found. This exponent corresponds to an unlimited diffusion on an infinite one-dimesional line which is in contrast to a bounded phase space. This strange observation was explained in [18, 19 as a diffusion in a chaotic separatrix layer of a nonlinear resonance which takes place on relatively short diffusion times. On larger times, which were not accessible to the computations presented in [17, this diffusion becomes bounded by a finite width of the separatrix layer and a universal algebraic decay takes place with the exponent $\beta \approx 1.5$ corresponding to a finite chaos measure [18,19]. This algebraic decay of $P(t)$ has been confirmed by various groups in various Hamiltonian systems [20,21], 22], 24, 25], 26, 27], [28, 29, 30, 31.

One can argue that such a slow algebraic decay with $\beta \approx 1.5$ appears due to trajectory sticking near stable islands and critical invariant curves and leads to an even slower correlation function decay $C(t) \sim t P(t)$ with a divergence of certain second moments. A sticking in a vicinity of the critical golden curve [10] is expected to give $\beta \approx 3[24,25$, being significantly larger than the average value $\beta \approx 1.5$. A certain numerical evidence is presented in [27] showing that long time sticking orbits can be trapped not only in a vicinity of a critical golden curve but also in internal chaotic layers of secondary resonances.

Theoretical attempts to describe trapping in secondary resonances as renormalization dynamics on some Cayley type tree was started in 22 with recent extensions done in $28,32,33$. However, a detailed understanding of the intriguing features of Poincaré recurrences in the Chirikov standard map and other similar maps is still missing.

In this work we use a generalized Ulam method developed in 34,35] and combine it with a new survival Monte Carlo method trying to reach larger time scales and to obtain a better understanding of statistics of Poincaré recurrences in the Chirikov standard map and the separatrix map.

The paper is composed as follows: in Section 2 we construct the Ulam matrix based on the generalized Ulam method and study the properties of its spectrum, eigenstates and corresponding time evolution for the case of the Chirikov standard map. The survival Monte Carlo method is introduced in Section 3 and the properties of the Poincaré recurrences are studied with its help compar- ing results with the Ulam method. In Section 4 we apply the above methods to the separatrix map and in Section 5 the localization properties of the eigenstates of the Ulam matrix are analyzed. The discussion of the results is presented in Section 6.

\section{Generalized Ulam method with absorption}

The Ulam method was proposed in 1960 [36]. In the original version of this method a $2 \mathrm{D}$ phase space is divided in $N_{d}=M \times M$ cells and $n_{c}$ trajectories are propagated on one map iteration from each cell $j$. Then the matrix $S_{i j}$ is defined by the relation $S_{i j}=n_{i j} / n_{c}$ where $n_{i j}$ is the number of trajectories arriving from a cell $j$ to a cell $i$. By construction we have $\sum_{i} S_{i j}=1$ and hence the matrix $S_{i j}$ belongs to the class of the Perron-Frobenius operators (see e.g. 37]). This Ulam matrix can be considered as a discrete Ulam approximate of the Perron-Frobenius operator (UPFO) of the continuous dynamics.
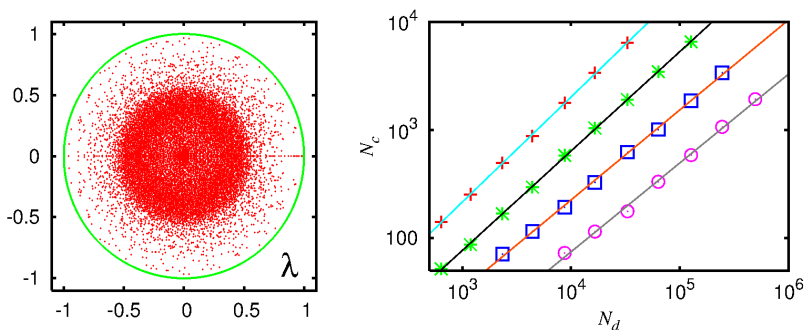

Fig. 1. (Color online) The left panel shows the eigenvalue spectrum $\lambda_{j}$ for the projected case of the UPFO of map (1) at $K=K_{g}$ in the complex plane for $M=280$ and $N_{d}=16609$ by red/gray dots (projected matrix dimension $N_{p}=15457$ ). The green/gray curve represents the circle $|\lambda|=1$. The right panel shows the number $N_{c}$ of eigenvalues, with modulus larger than $\lambda_{c}$, versus $N_{d}$ in a double logarithmic representation for $\lambda_{c}=0.5$ (crosses), $\lambda_{c}=0.66$ (stars), $\lambda_{c}=0.8$ (open squares) and $\lambda_{c}=0.9$ (open circles). The straight lines correspond to the power law fits $N_{c} \sim N_{d}^{\nu}$ with exponents $\nu=0.971 \pm 0.006$ $\left(\lambda_{c}=0.5\right), \nu=0.919 \pm 0.005\left(\lambda_{c}=0.66\right), \nu=0.832 \pm 0.010$ $\left(\lambda_{c}=0.8\right)$ and $b=0.821 \pm 0.021\left(\lambda_{c}=0.9\right)$. The fits are done for the data with $N_{c}>50, M>35$ and $M \leq 400\left(\lambda_{c}=0.5\right)$, $M \leq 800\left(\lambda_{c}=0.66\right), M \leq 1120\left(\lambda_{c}=0.8\right), M \leq 1600\left(\lambda_{c}=\right.$ $0.9)$, since the Arnoldi method provides only a partial spectrum of the eigenvalues with largest modulus for large values of $M$.

According to the Ulam conjecture [36] the UPFO converges to the continuous limit at large $M$. Indeed, this conjecture was proven for 1D homogeneously chaotic maps 38. Various properties of the UPFO for $1 \mathrm{D}$ and $2 \mathrm{D}$ maps are analyzed in 39, 40, 41,42. Recent studies 44, 45] demonstrated similarities between the UPFO, the corresponding to them Ulam networks and the properties of the Google matrix of the world wide web networks. It was shown that in maps with absorption or dissipation the spectrum of the UPFO is characterized by the fractal Weyl law [46.

The coarse-grained cell structure of the original Ulam method corresponds to an effective noise and in case of 

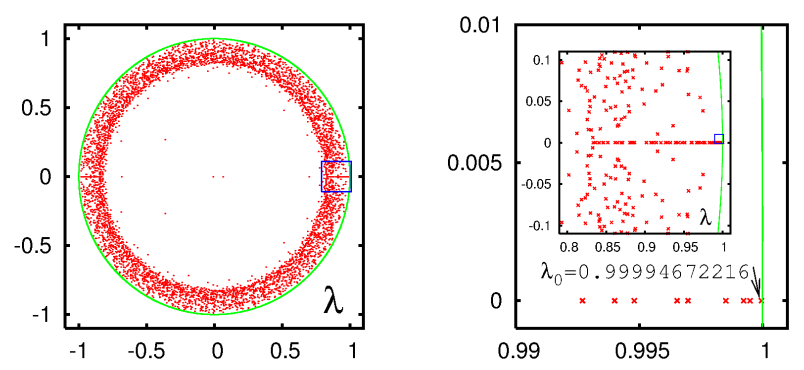

Fig. 2. (Color online) Partial spectrum $\lambda_{j}$ for the projected case of the UPFO of the map (1) at $K=K_{g}$ for $M=1600$. The left panel shows all eigenvalues obtained by the Arnoldi method with $n_{\mathrm{A}}=5000$. The insert of the right panel shows the blue/black square of the first zoomed range of the left panel; the blue/black square here is the second zoomed range shown in the main figure of the right panel. The eigenvalue with largest modulus $\lambda_{0}=0.99994672216$ is indicated by an arrow. The green/gray curve represents in all cases the circle $|\lambda|=1$.

a divided phase space the noise induces an artificial diffusion between chaotic and regular regions. In 34 this problem was solved by replacing the random initial points by a very long chaotic trajectory and the transitions between cells are accumulated along the chaotic trajectory that keeps the invariant curves and stable islands even in presence of the effective noise. Furthermore, the matrix size is also reduced since only cells which are visited at least once by the trajectory are kept. Here we use this approach for the analysis of the Poincaré recurrences keeping the same notations as in 34]. In particular, as in 34], we exploit the parity symmetry $x \rightarrow 1-x$ and $y \rightarrow 1-y$ allowing to limit the effective phase space to $0 \leq x \leq 1$, $0 \leq y \leq 0.5$ and therefore reducing the number of cells at a given cell size by a factor of two. In $x$ direction we use therefore $M$ cells and in $y$ direction $M / 2$ cells with $M \in\{25,35, \ldots, 1120,1600\}$ and the intermediate values are multiples of 25 or 35 by powers of 2 .

To study the Poincaré recurrences withing the Ulam method we introduce absorption of all trajectories with $y<y_{\text {cut }}=0.05$. Thus we generate the matrix $S$ using one trajectory iterated by the map up to the iteration time $t=10^{12}$ (as in [34; this corresponds to the closed system without absorption and we call this the symplectic case). After that the matrix size $N_{d}$ is simply reduced only to those cells with $y \geq y_{\text {cut }}$ that gives the projected matrix dimension $N_{p}$ and matrix $S_{p}$. The matrix size of this projected case is smaller approximately by $7 \%$. We find, for $M \leq 1600$, an approximate dependence $N_{d} \approx 0.39 M^{2} / 2$ and $N_{p} \approx 0.36 M^{2} / 2$. This corresponds to the usual estimate of the chaos measure being around $39 \%$ in agreement with the results of Chirikov [6]. For the symplectic case we have the maximal eigenvalue $\lambda=1$ while in the projected case with absorption we are getting $|\lambda|<1$.

The spectrum $\lambda_{j}$ of the projected case with matrix $S_{p}$ is shown in Fig. 1. The spectrum is obtained by the direct diagonalization of the matrix $S_{p}$ that can be done numerically up to $M=280$. It can be compared with the corresponding spectrum of the symplectic system shown in Fig. 2 of [34]. The global spectrum structure of $S$ for the symplectic case is similar to the projected case. Indeed, the absorption is relatively weak and does not affect the global properties of motion. However, with absorption the measure is not conserved and the remaining non-escaping set forms a fractal set with the fractal dimension $d<2$ (see e.g. 46, 47]).

In the case of Ulam networks on fractal chaotic repellers the spectrum of UPFO $S_{p}$ is characterized by the fractal Weyl law with the number of states $N_{c}$ in the ring $\lambda_{c}<|\lambda| \leq 1$ growing with the matrix size $N_{d}$ as $N_{c} \propto N_{d}^{\nu}$ (here for simplicity we use the size $N_{d}$ of the symplectic case, for the projected case we have simply to change $N_{p} \approx 0.93 N_{d}$ ). It can be argued that the fractal dimension $d_{0}$ of the invariant repeller set determines the exponent $\nu=d_{0} / 2$ [46]. Examples of dependencies $N_{c}$ vs $N_{d}$ are given in Fig. 1 for various values of $\lambda_{c}$. Definitely we have $\nu<1$ but there is an evident dependence on $\lambda_{c}$ with a decreasing value of $\nu$ at $\lambda_{c} \rightarrow 1$. We attribute this to the fact that at $\lambda_{c} \rightarrow 1$ we are dealing with long sticking trajectories whose measure decreases with time.
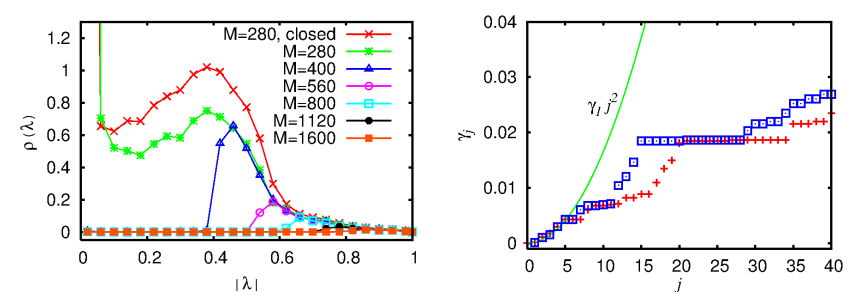

Fig. 3. (Color online) The left panel shows the density $\rho(\lambda)$ of eigenvalues, being normalized by $\int \rho(\lambda) d^{2} \lambda=1$, of the UPFO for the map (1) at $K=K_{g}$ in the complex plane as a function of the modulus $|\lambda|$ for $M=280$ for the symplectic case (upper curve, crosses) and the projected case (lower green curve, stars). The other curves are partial (non-normalized) densities for the projected case and the values $M=400,560,800,1120,1600$ and the number of used eigenvalues (obtained by the Arnoldi method) is $n_{A}=12000,8000,8000,6000,5000$ respectively. The right panel shows the decay rates $\gamma_{j}=-2 \ln \left(\left|\lambda_{j}\right|\right)$ versus level number $j$ for the UPFO eigenvalues $\lambda_{j}$, with $M=1600$ and $N_{d}=494964$. The red/gray crosses correspond to the UPFO of symplectic case and the blue/black squares correspond to the projected case (data points for this case are shifted to one position to the right). The green curve corresponds to the quadratic dispersion law $\gamma_{j} \approx \gamma_{1} j^{2}$ which is approximately valid for the diffusion modes with $0 \leq j \leq 5$ and where $\gamma_{1}$ is taken from the UPFO of the symplectic case.

Here we should point out that the data for $M \geq 400$ corresponding to $N_{d}>30000$ are obtained from the Arnoldi method [48] which allows to find the eigenvalues for matrix sizes up to $N_{d} \sim 10^{6}$. However, only a finite number of eigenvalues with largest $|\lambda|$ can be determined numerically using $n_{A}=12000,8000,8000,6000,5000$ (for $M=400,560,800,1120,1600$ respectively and with $n_{A}$ being the used Arnoldi dimension). A more detailed de- 
scription of the Arnoldi method for the UPFO is given in [34. An example of the spectrum $\lambda$ obtained with the Arnoldi method at the largest value of $M=1600$ is shown in Fig. 2. Here $N_{d}=494964$ and $N_{p}=458891$. We find that the maximal eigenvalue for the projected case is $\lambda_{0}=0.99994672216$ corresponding to a slow escape rate at large times. As in 34 for the symplectic case without absorption, we obtain also for the case with absorption two type of eigenmodes: "diffusion modes" with real eigenvalues close to 1 and whose eigenvectors are rather extended in phase space (with some decay for cells close to the absorption border) and "resonant modes" with complex or real negative eigenvalues and which are quite well localized around a chain of stable islands close to an invariant curve. It turns out that many of the resonant modes (those "far" away from the absorption border), coincide numerically very well with corresponding resonant modes for the case without absorption already found in 34 .

The dependence of the density of eigenvalues $\rho(|\lambda|)$ on $|\lambda|$ is shown in Fig. 3. We see the proximity between the symplectic and projected cases not only in density $\rho$ but also in a slow relaxation of the diffusion modes with relaxation rates $\gamma_{j} \approx \gamma_{1} j^{2}\left(\gamma_{j}=-2 \ln \left|\lambda_{j}\right|\right)$ provided we identify $\gamma_{j+1}$ of the symplectic case with $\gamma_{j}$ of the projected case because $\gamma_{0}$ of the symplectic case is simply zero and the relaxation rate $\gamma_{1}$ to the ergodic state of the symplectic case corresponds roughly to the exponential long time escape rate $\gamma_{0}$ of the projected case. The proximity of the two cases is also well seen in the dependence of integrated density of states $\rho_{\Sigma}(\gamma)=j / N_{d}$ on $\gamma_{j}$ shown in Fig. 4 (here $j$ is a number of eigenvalues with $\gamma \leq \gamma_{j}$ ). In both cases we have the algebraic dependence $\rho_{\Sigma}(\gamma) \propto \gamma^{\beta}$ with $\beta \approx 1.5$. In 34 it was argued that this exponent is the same as for the exponent of decay of Poincaré recurrences $P(t)$. These data show that an introduction of small absorption at $y<y_{\text {cut }}$ does not produce significant modification for trajectories trapped for long times in a vicinity of the critical golden curve or other secondary islands located far away from the absorption band.

The lowest eigenvalues such like $\gamma_{0}$ and $\gamma_{5}$ decrease algebraically with the increase of $M$ as it is shown in right panel of Fig. 4. In the fit range $400 \leq M \leq 1600$ we have a power law $\gamma_{0}(M) \approx 0.72 M^{-1.20}$ but taking into account the curvature for the interval $25 \leq M \leq 1600$ the modified fit $\gamma_{0}(M)=\frac{D}{M} \frac{1+C / M}{1+B / M}$ with $D=0.162, C=165$ and $B=$ 17.0 seems to indicate a behavior $\gamma_{0}(M) \propto M^{-1}$ in the limit $M \rightarrow \infty$. This behavior is similar to the one found in [34] for $\gamma_{1}$ in the symplectic case (where $\gamma_{0}$ is simply 0 ). On the other hand the resonant mode $\gamma_{5}$ obeys the power law $\gamma_{5}(M) \approx 389 M^{-1.55}$ which is valid for the interval $100 \leq M \leq 1600$ if we use for the smaller values of $M$ not $\gamma_{5}$ but the resonant mode localized to the same chain of resonant islands which may have a different eigenvalue index (see Fig. 4 for details). The comparison of these decays indicate that eventually at very large values of $M$ far outside the range numerically accessible by the Arnoldi method, the resonant modes become dominant over the diffusion modes. The limit $\gamma \rightarrow 0$ for $M \rightarrow \infty$ is related to long sticking trajectories near critical invariant curves
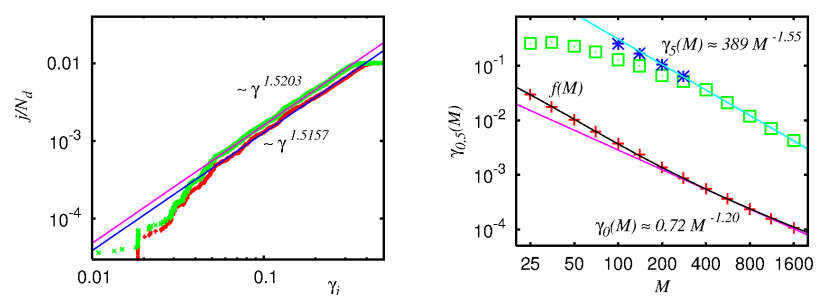

Fig. 4. (Color online) The left panel shows the rescaled level number $j / N_{d}$ versus the decay rate $\gamma_{j}$, in a double logarithmic scale, for the map (1) at $K_{g}$ with $M=1600$ and $N_{d}=494964$. Red/lower data points correspond to the UPFO projected case and green/upper data points correspond to the UPFO symplectic case. The two straight lines correspond to the power law fits $j / N_{d} \approx 0.052745 \gamma^{1.5203}$ (symplectic case) and $j / N_{d} \approx$ $0.041570 \gamma^{1.5157}$ (projected case) for the data in the range $0.04 \leq \gamma \leq 0.3$. The statistical error bound of the exponents obtained from the fits is close to $0.1 \%$ in both cases. The right panel shows the decay rates $\gamma_{j}(M)$ for $j=0$ (red crosses), $j=5$ (green open squares) of the UPFO projected case in a double logarithmic scale. The lower/pink straight line corresponds to the power law fit $\gamma_{0}(M) \approx 0.72 M^{-1.20}$ and the upper/light blue straight line to the fit $\gamma_{5}(M) \approx 389 M^{-1.55}$ (both fits obtained for the range $400 \leq M \leq 1600)$. The black/curved line corresponds to the other fit $\gamma_{0}(M)=f(M)=\frac{D}{M} \frac{1+C / M}{1+B / M}$ with $D=0.162, C=165$ and $B=17.0$ (fit obtained for the range $25 \leq M \leq 1600)$. We mention that $\gamma_{5}$ corresponds for $M \geq 400$ to a resonant mode whose eigenvector is strongly localized close to the three stable islands of the resonance $1 / 3$. However, for $M \leq 280 \gamma_{5}$ corresponds to a different mode and the resonant mode at $1 / 3$ is associated to $\gamma_{7}(M=280), \gamma_{13}$ $(M=200), \gamma_{17}(M=140)$ and $\gamma_{23}(M=100)$ which are shown as four additional data points (blue stars).

which restrict the chaos component and whose phase space structure can be better resolved with decreasing cell size $1 / M$. As in 34 we argue that these lowest modes are affected by the effective noise present in the Ulam method. Due to that we do not have a clear explanation for this algebraic decay. However, the fact that $\gamma_{j}$ (at fixed value of $j$ ) vanishes with increasing $M$ indicates that the limit $t_{\exp }$ in time, when the statistics of Poincaré recurrences $P(t)$ obtained from the UPFO becomes exponential, increases as well according to $t_{\exp } \propto \gamma_{0}^{-1}$ and therefore we expect to recover the power law decay of $P(t)$ for $M \rightarrow \infty$ (see below in Section 3).

With the help of the Arnoldi method we find certain eigenstates corresponding to eigenvalues of the matrix $S_{p}$ and satisfying the equation

$$
\sum_{i=0}^{N_{p}-1}\left(S_{p}\right)_{m i} \psi_{j}(i)=\lambda_{j} \psi_{j}(m)
$$

Examples of two eigenmodes $\left|\psi_{0}\right|$ and $\left|\psi_{29}\right|$ are shown in Fig. 5. The state $\left|\psi_{0}\right|$ corresponds to the first diffusive mode mainly located in a vicinity of the critical golden curve while $\left|\psi_{29}\right|$ corresponds to the mode located near a resonant chain with rotation number $r=2 / 7$. 

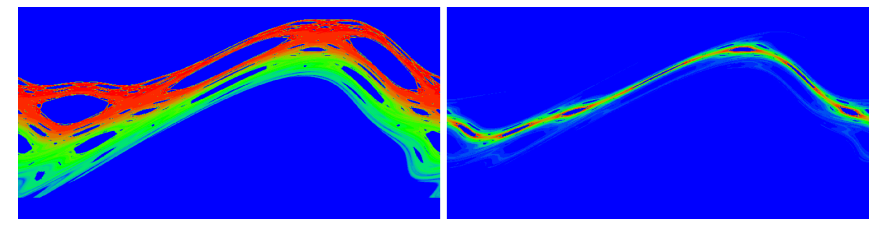

Fig. 5. (Color online) Density plot of the modulus of the eigenvector components $|\psi|$ of the UPFO projected case of map (1) at $K_{g}$ with $M=1600$ for the two modes with eigenvalues $\lambda_{0}=0.99994672$ (left panel) and $\lambda_{29}=-0.22008951+$ $i 0.96448508 \approx\left|\lambda_{29}\right| e^{i 2 \pi(2 / 7)}$ (right panel). The density is shown by color with red/gray for maximum and blue/black for zero.
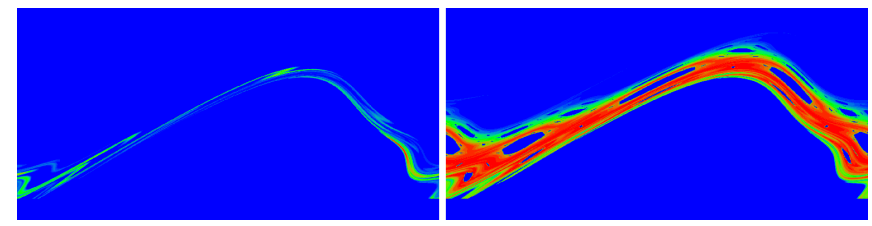

Fig. 6. (Color online) Time dependent probability density calculated by $\psi(t)=\left(S_{p}\right)^{t} \psi(0) /\left\|\left(S_{p}\right)^{t} \psi(0)\right\|_{1}$ where $S_{p}$ is the UPFO for the projected case for $M=1600, \psi(0)$ an initial vector with $\psi_{l}(0)=\delta_{l, \ell_{0}}$ and $\ell_{0}$ being the index of the cell at $x_{0}=y_{0}=0.0625$ and $\|\cdots\|_{1}$ is the 1 -norm defined by $\|\psi\|_{1}=\sum_{l}\left|\psi_{l}\right|$. The densities are shown for $t=40$ (left panel) and $t=400$ (right panel). In the limit $t \rightarrow \infty$ the vector $\psi(t)$ converges to the eigenvector of maximal eigenvalue $\lambda_{0}$ shown in the left panel of Fig. 5 The full convergence is achieved for $t \geq 40000$ so that for these times the density plot of $\psi(t)$ remains unchanged at the given color-resolution.

It is also interesting to follow how the probability initially placed in one cell $\ell_{0}$ evolves with time. Of course, the total probability starts to decay due to absorption but by renormalizing the total probability back to unity after each map iteration we obtain its evolution in phase space. At large times we have convergence to the state $\psi_{0}$ with maximal $\lambda_{0}$ but at intermediate times we see the regions of phase space which contribute to long time sticking and long Poincaré recurrences. Two snapshots are shown in Fig. 6. The videos of such an evolution for the maps (1) and (3) are available at 35 .

\section{Poincaré recurrences by survival Monte Carlo method}

The numerical computation of the Poincaré recurrences counting the number of crossing of a given line (e.g. $y=0$ ) in the phase space is known to be a very stable numerical method since the integrated probability of recurrences on a line at times larger than $t$ is positively defined (see e.g. [18, 19, 24, 28] ). However, at large times the direct numerical computation becomes time consuming.

With the aim to reach larger times we present here a new method to calculate the statistics of Poincaré recurrences of map such as the Chirikov standard map (1).
We will call this method the Survival Monte Carlo method (SMCM). The idea of this method is to chose a certain, quite large number $N_{i} \gg 1$, of initial conditions randomly chosen in some small cell close to an unstable fix point and to calculate in parallel the time evolution of these trajectories. At the initial time $t=0$ we put the Poincaré return probability to $P(0)=1$ and the number of trajectories to $N(0)=N_{i}$. At each time $t_{k}$, when a given trajectory escapes in the absorption region $y<y_{c u t}=0.05$ of the phase space, we put $P\left(t_{k}+1\right)=P\left(t_{k}\right)\left(N\left(t_{k}\right)-1\right) / N\left(t_{k}\right)$ and $N\left(t_{k}+1\right)=N\left(t_{k}\right)-1$, otherwise we simply keep $P\left(t_{k}+1\right)=P\left(t_{k}\right)$ and $N\left(t_{k}+1\right)=N\left(t_{k}\right)$. When the number of remaining trajectories $N\left(t_{k}\right)$ drops below a certain threshold value $N_{f}$ (typically chosen such that $N_{i} \gg N_{f} \gg 1$ ) we reinject a new trajectory close to one of the other remaining trajectories with a small random deviation: $x_{\text {new }}(t) \in\left[x_{i}(t)-\varepsilon / 2, x_{i}(t)+\varepsilon / 2\right]$ and $y_{\text {new }}(t) \in\left[y_{i}(t)-\varepsilon / 2, y_{i}(t)+\varepsilon / 2\right]$. The main idea is to keep a typical statistics of trajectories at a given time $t$ and to concentrate the computational effort on the very long and rare trajectories without wasting resources on the more probable trajectories with short times of Poincaré recurrences.
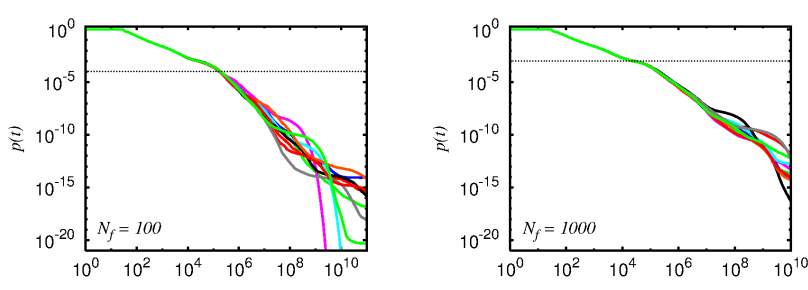

Fig. 7. (Color online) Statistics of Poincaré recurrences $P(t)$ of the map (1) calculated by the SMCM as survival probability after times larger than $t$ (data are shown in double logarithmic scale). The number of initial trajectories is $N_{i}=10^{6}$ and the number of final trajectories is $N_{f}=100$ (left panel) or $N_{f}=1000$ (right panel). The initial positions are randomly chosen in a cell of size $(1600)^{-1} \times(1600)^{-1}$ at the position $x_{0}=y_{0}=0.0625$, here the small random deviation for reinjected trajectories is $\sim \varepsilon=10^{-14}$. In both panels the results for $P(t)$ are shown for 10 realizations with different random seeds. The horizontal dotted line indicates the limit probability $N_{f} / N_{i}=10^{-4}$ (left panel) or $N_{f} / N_{i}=10^{-3}$ (right panel) below which the reinjection of trajectories is applied. The two realizations in the left panel which drop below the shown range (of $P(t) \geq 10^{-21}$ ) "saturate" eventually at the values $P\left(10^{11}\right) \approx 2 \times 10^{-36}$ or $P\left(10^{11}\right) \approx 10^{-35}$.

In this method the proper choice of $\varepsilon$ is important. On one hand $\varepsilon$ should not be too small in order to avoid too strong correlations between the trajectories and on the other hand it should be very small in order to avoid an uncontrolled too strong diffusion into regions too close to stable islands where the trajectories may be trapped stronger and longer as they should be without the random deviations. Fortunately in the chaotic region even a modest Lyapunov exponent ensures exponential separation of 
trajectories and choosing a very small value of $\varepsilon$ one may hope to reduce the correlation between the injected trajectory and its reference trajectory after a modest number of iterations. Furthermore at longer times the average time between the escape of two trajectories becomes very large that helps to reduce these correlations.
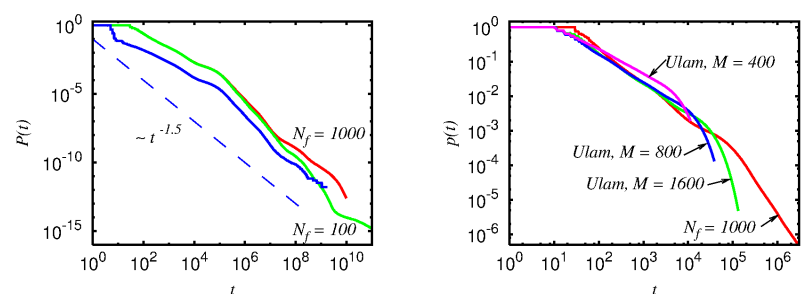

Fig. 8. (Color online) The left panel shows the average over 10 random realizations of the statistics of Poincaré recurrences $P(t)$ of the map (1), obtained by the SMCM for survival probability and shown in Fig. 7. The upper/red curve, for $t \leq 10^{10}$, corresponds to $N_{f}=1000$. The next lower/green curve, for $t \leq 10^{11}$, corresponds to $N_{f}=100$. The lowest blue curve, for $t \leq 1.7 \times 10^{9}$, corresponds to the data of Refs. 25,26 , obtained by a direct computation of the statistics of Poincaré recurrences. The dashed straight line indicates a power law behavior $P \propto t^{-1.5}$. The right panel compares the statistics of Poincaré recurrences $P(t)$ obtained by the SMCM for $N_{f}=1000$ to $P(t)$ obtained by the Ulam method for $M=400,800,1600$. At large times $t>t_{\exp } \sim 10^{4}-10^{5}$ the curves obtained by the Ulam method show an exponential behavior $P(t) \sim \lambda_{0}^{t}$ determined by the largest eigenvalue of the UPFO for the projected case

We have chosen the parameters $\varepsilon=10^{-14}, N_{i}=10^{6}$ and the two cases $N_{f}=100$ and $N_{f}=1000$. For $N_{f}=100$ we have been able to iterate up to times $10^{11}$ and for $N_{f}=1000$ up to times $10^{10}$. We mention that at the "larger" value $\varepsilon=10^{-10}$, we observe a significant effect of artificial saturation in $P(t)$ (i. e. no escapes of trajectories) at longer times because the trajectories penetrate "too strongly" into the regions very close to the stable islands or the critical curve. When we choose $\varepsilon=10^{-14}$ this artificial saturation effect is strongly reduced. We calculate in parallel different realizations of $P(t)$ with respect of the random variables (for the initial conditions, for the random deviations of the reinjected trajectories and for the random choice at which remaining trajectory the reinjection happens). The comparison of obtained data shows that the distribution $P(t)$ is stable at small and large times. But at very large times it turns out that the fluctuations become quite strong.

Examples of the survival probability $P(t)$ obtained for 10 different realizations with $N_{f}=100$ (left panel) and $N_{f}=1000$ (right panel) are shown in Fig. 7. Of course the fluctuations appear for $N_{f}=100$ at shorter times $\left(t \sim 10^{5}-10^{6}\right)$ as compared to $N_{f}=1000\left(t \sim 10^{6}-10^{7}\right)$.

We note that the SMCM allows us to determine the survival probability $P(t)$. Its comparison with the statistics of Poincaré recurrences computed by the usual method
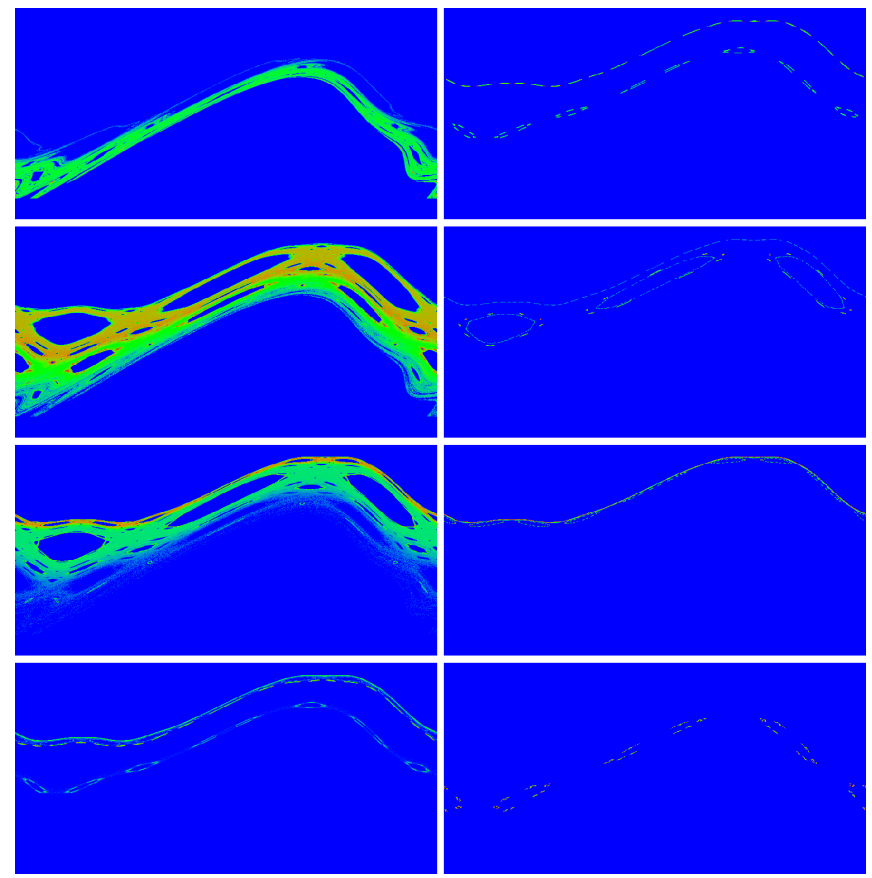

Fig. 9. (Color online) Density plots of the trajectories of the SMCM (with $N_{f}=1000$ ) for the map (1) for various times $t$ and random realizations. All density plots are obtained from a histogram of $10^{7}$ data points and using a resolution of $800 \times 400$ cells for the phase space $0 \leq x<1$ and $0 \leq y<0.5$. The data points are obtained by iterating $N(t)$ trajectories (with $N(t)=P(t) N_{i}$ for $P(t) \geq 10^{-3}$ and $N(t)=N_{f}$ for $\left.P(t)<10^{-3}\right)$ from $t$ to $t+\Delta t$ with $\Delta t=10^{7} / N(t)$. The left four panels and the upper right panel correspond to one particular random realization at $t=10^{2}, 10^{4}, 10^{6}, 10^{8}, 10^{10}$ and the three lower right panels correspond to three other random realizations at $t=10^{10}$. For short times $t<10^{5}$ there is no significant difference between the density plots for different random realizations at a given time. More detailed density plots for intermediate times and higher resolution figures are available at 35 .

18, 24, 25, 26 is shown in Fig. 8. We see that both methods give the same behavior $P(t)$ with a small shift in time related to different initial conditions. The equivalence of both methods is rather clear: in both methods the probability is determined by long sticking trajectories; both methods consider the recurrences to the lines $y=0$ or $y=0.05$ which are close to each other.

The decay of $P(t)$ averaged over 10 random realizations is shown in Fig. 8. In general we see that the SMCM allows to reach extremely long times with $t=10^{11}$ for $N_{f}=100$ and $t=10^{10}$ for $N_{f}=1000$. For $N_{f}=100$ we see that the fluctuations start to be important for $t>10^{9}$ while the case with $N_{f}=1000$ remains stable up to $t=10^{10}$. This allows to obtain the behavior of $P(t)$ for times being about one order of magnitude larger compared to previous numerical simulations.

For the case $N_{f}=1000$ in Fig. 8 the algebraic fit of data in the range $10^{6} \leq t \leq 10^{10}$ gives the Poincaré exponent $\beta=1.587 \pm 0.009$. For $N_{f}=100$ case we find 
$\beta=1.710 \pm 0.017$ for the range $10^{6} \leq t \leq 10^{11}$. The formal statistical error is rather small in both cases but it is clear that for $N_{f}=100$ we start to have an effect of strong fluctuations due to long sticking around islands and thus the reliable value of $\beta$ is given by the case with $N_{f}=1000$.

The survival probability $P(t)$ can be also computed using the Ulam method at various sizes of discrete cells determined by $M$. The results obtained by the generalized Ulam method and by the SMCM are shown in the right panel of Fig. 8, The comparison shows that both methods give the same results but the SMCM is much more efficient allowing to follow the decay $P(t)$ up to significantly larger times since for the Ulam method we expect the decay $P(t)$ only to be accurate for $t<t_{\exp } \sim \gamma_{0}^{-1}$ because for $t>t_{\exp }$ it becomes exponential $P(t) \propto \lambda_{0}^{t}=\exp \left(-\gamma_{0} t / 2\right)$. The data of Fig. 8 clearly shows that $t_{\text {exp }}$ increases with $M$ in accordance with the decay of $\gamma_{0}$ obtained from Fig. 4

Using the SMCM we can follow the evolution of the survival probability as a function of time showing the density plot of long sticking trajectories. Examples of such distributions are shown in Fig. 9. These Figs. show that at short times $t<100$ the trajectories are not yet able to cross the cantori barriers and remain relatively far from the golden curve, at larger times $t=10^{4}, 10^{6}, 10^{8}$ the probability becomes concentrated close to the golden curve. But at very larger times $t=10^{10}$ we find trajectories sticking in a vicinity of the golden curve or other secondary resonances. Thus we see that at long time $P(t)$ has contributions not only from the vicinity of the critical golden curve but also from other secondary resonances. In this respect, our conclusion confirms a similar one expressed in 27] obtained from simulations on shorter time scales.

\section{Separatrix map with critical golden curve}

To show that the previous case of the Chirikov standard map represents a generic situation we also study the UPFO of the projected case for the separatrix map [6], defined by :

$$
\bar{y}=y+\sin (2 \pi x), \quad \bar{x}=x+\frac{\Lambda}{2 \pi} \ln (|\bar{y}|)(\bmod 1) .
$$

This map can be locally approximated by the Chirikov standard map by linearizing the logarithm near a certain $y_{0}$ that leads after rescaling to the map (1) with an effective parameter $K_{\text {eff }}=\Lambda /\left|y_{0}\right|$ [6]. As in [34] we study the map (3) at $\Lambda_{c}=3.1819316$ with the critical golden curve at the rotation number $r=r_{g}=(\sqrt{5}-1) / 2=0.618 \ldots$ The construction of the matrix $S$ is described in 34 , its size is given by an approximate relation $N_{d} \approx 0.78 M^{2} / 2$ for the phase space region $0<x \leq 1,0 \leq y \leq 4$ (symplectic case and using the symmetry: $x \rightarrow x+1 / 2(\bmod 1), y \rightarrow-y)$. The absorption is done for $y<y_{\text {cut }}=0.4$ corresponding to $10 \%$ of the maximal possible value of $y$. Thus for the UPFO for the projected case we have $N_{p} \approx 0.68 M^{2} / 2$. In fact we have $2\left(N_{d}-N_{p}\right) / M^{2}=0.1$ since all part of the phase space is chaotic at $0<y<y_{\text {cut }}$ and all cells in this region were occupied by the Ulam method. Thus for $M=1600$ we have $N_{d}=997045, N_{p}=869045$.
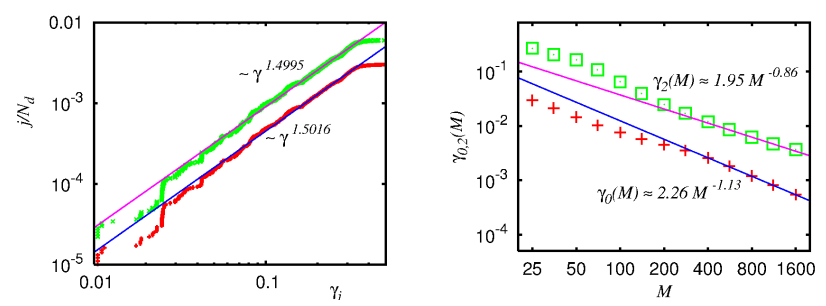

Fig. 10. (Color online) The left panel shows the rescaled level number $j / N_{d}$ versus the decay rate $\gamma_{j}$, in a double logarithmic scale, for the separatrix map (3) at $\Lambda_{c}$ with $M=1600$ and $N_{d}=997045$. Red/lower data points correspond to the UPFO for the projected case and green/upper data points correspond to the symplectic case. For the symplectic case the data points are shifted up by a factor 2 to separate the two data sets. The two straight lines show the power law fits $j / N_{d} \approx$ $0.014173 \gamma^{1.4995}$ (symplectic case) and $j / N_{d} \approx 0.014207 \gamma^{1.5016}$ (projected case) for the range $0.04 \leq \gamma \leq 0.3$. The statistical error of the exponents is close to $0.2 \%$ in both cases. The right panel shows the decay of $\gamma_{j}(M)$ with $M$ for $j=0$ (red crosses), $j=2$ (green open squares) for the UPFO for the projected case of map (3). The lower/blue straight line corresponds to the power law fit $\gamma_{0}(M) \approx 2.26 M^{-1.13}$ and the upper/pink straight line to the fit $\gamma_{2}(M) \approx 1,95 M^{-0.86}$ (for the range $400 \leq M \leq 1600)$. The eigenvector corresponding to $\gamma_{2}$ is localized near the two stable islands of the resonance $1 / 2$.

In Fig. 10, in analogy to Fig. 4, we show the dependence of integrated number of eigenvalues $j / N_{d}$ on $\gamma_{j}=$ $-2 \ln \left|\lambda_{j}\right|$ for the symplectic and projected cases of the UPFO of the map (3). In both cases we have approximately the same dependence with the algebraic exponent $\beta \approx 1.5$ which works for the range $0.04 \leq \gamma \leq 0.3$. The minimal values of $\gamma$ (e.g. $\gamma_{0}$ and $\gamma_{2}$ ) drop approximately inversely proportionally to $M$. As for symplectic case 34 we attribute this decrease with $M$ to a finite size coarsegraining effect of the Ulam method. As in 34, we argue that the exponent $\beta$ for a more physical intermediate range of $\gamma$ is directly related to the Poincaré exponent.
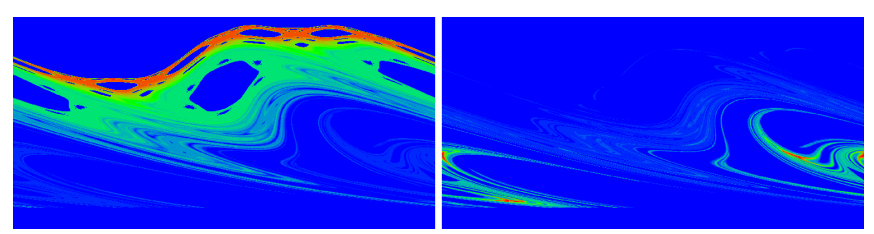

Fig. 11. (Color online) Density plot of the modulus of the eigenvector components of the UPFO for the projected case of the map (3) at $M=1600$ for the two modes with $\lambda_{0}=0.99972660$ (left panel) and $\lambda_{77}=-0.49158775+$ $i 0.85153885 \approx\left|\lambda_{77}\right| e^{i 2 \pi(1 / 3)}$ (right panel). 
Examples of two eigenmodes at $\lambda_{0}$ and $\lambda_{77}$ are shown in Fig. 11. In the first case we have an eigenmode of diffusive type similar to Fig. 5 while in the latter case we have an eigenmode concentrated around unstable fix points of resonance $1 / 3$ (see corresponding state of symplectic case in bottom left panel of Fig.11 in [34]).
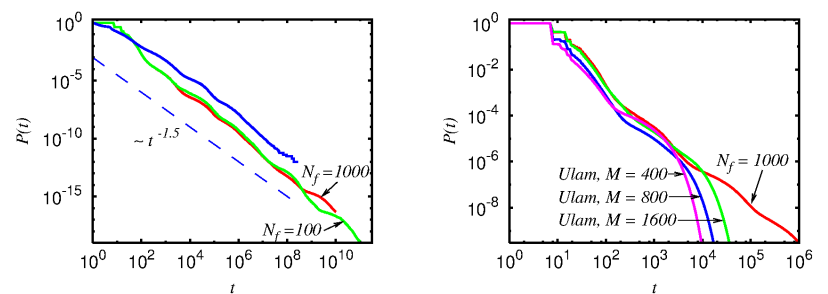

Fig. 12. (Color online) The left panel shows the average over 10 random realizations of the statistics of Poincaré recurrences $P(t)$ of the map (3), obtained by the SMCM. The red curve, for $t \leq 10^{10}$, corresponds to $N_{f}=1000$. The green curve, for $t \leq 10^{11}$, corresponds to $N_{f}=100$. The upper/blue curve, for $t \leq 2.8 \times 10^{8}$, corresponds to the data shown in 25 using a direct computation of the statistics of Poincaré recurrences. The right panel compares $P(t)$ SMCM data for $N_{f}=1000$ (red curve in left and right panels) with $P(t)$ obtained by the Ulam method for $M=400,800,1600$. At large times $t>t_{\exp } \sim$ $2 \times 10^{3}-2 \times 10^{4}$ the Ulam method leads to an exponential decay $P(t) \sim \lambda_{0}^{t}$ determined by the largest eigenvalue of the UPFO for the projected case.

The comparison of the statistics of Poincaré recurrences obtained from the map (3) by the SMCM and the usual method are shown in Fig. 12, The data of the usual method obtained in [25] allows to follow the decay of $P(t)$ up to $t=2 \times 10^{8}$, while with the SMCM we reach times $t=10^{10}$ with $N_{f}=1000$ and $t=10^{11}$ with $N_{f}=100$. We have a good agreement between three curves for the range $100 \leq t \leq 10^{8}$ with a certain constant displacement in $\log _{10} t$ of data from the usual method compared to the SMCM data. This shift appears due to different initial conditions but apart of this shift all oscillations of $P(t)$ curve are well reproduced. This shows that both methods works correctly. However, with the SMCM we are able to reach times being by one to two orders of magnitude larger than previously.

The algebraic fit of SMCM data in Fig. 12 gives $\beta=$ $1.855 \pm 0.004$ for $N_{f}=100$ (range $10^{4} \leq t \leq 10^{11}$ ) and $\beta=1.706 \pm 0.004$ for $N_{f}=1000$ (range $10^{4} \leq t \leq 10^{10}$ ). In both cases the statistical error is rather small but there are visible fluctuations which become to be significant at $t>10^{9}$ for $N_{f}=100$ even if they are smaller compared to the similar case of map (1) shown in Fig. 8. Due to that one should take as the reliable value $\beta=1.706$ that shows a noticeable difference from the value $\beta=1.587$ found above for the Chirikov standard map at $K=K_{g}$.

The comparison of the SMCM data for $P(t)$ with the results of the Ulam method are shown in the right panel of Fig. 12. As it was the case for the similar comparison shown in Fig. 8 we find that both methods give the same
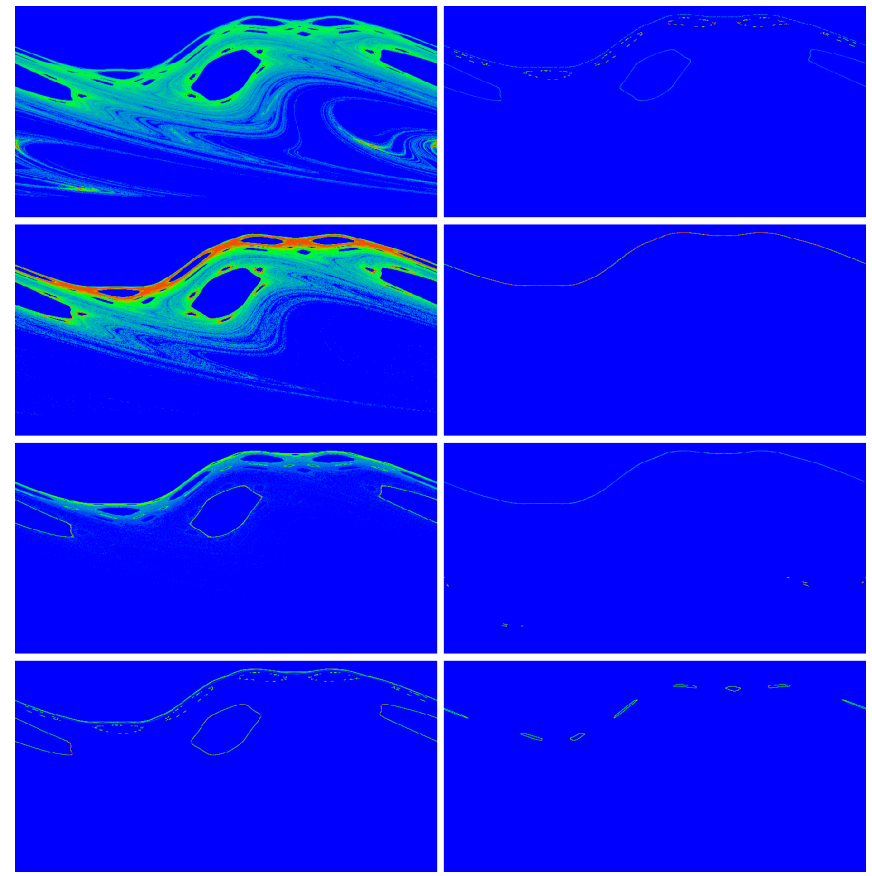

Fig. 13. (Color online) Density plots of the trajectories of the SMCM with $N_{f}=1000$ for the map (3) for various times $t$ and various realizations. All density plots are obtained by a histogram of $10^{7}$ data points with a resolution of $800 \times 400$ cells for the phase space $0 \leq x<1$ and $0 \leq y<4$. The data points are obtained by iterating the $N(t)$ trajectories (with $N(t)=P(t) N_{i}$ for $P(t) \geq 10^{-3}$ and $N(t)=N_{f}$ for $\left.P(t)<10^{-3}\right)$ from $t$ to $t+\Delta t$ with $\Delta t=10^{7} / N(t)$. The left four panels and the upper right panel correspond to one particular random realization at $t=10^{2}, 10^{4}, 10^{6}, 10^{8}, 10^{10}$ and the three lower right panels correspond to three other random realizations at $t=10^{10}$. For short times $t<10^{5}$ there is no significant difference between the density plots for different random realizations at a given time.

results but the Ulam method works only for time scales being significantly smaller than those reached with the SMCM.

Finally, as in Fig. 9, we show in Fig. 13 the density distribution obtained for various realizations and various times of the map (3). The situation is similar to Fig. 9 at short times the density is bounded by cantori barriers, at large times it reaches the critical golden curve and at even larger times we see that the density is located near the critical golden curve or other secondary resonances depending on the realization.

\section{Properties of eigenstates of Ulam matrix}

Let us now try to analyze how the decay of Poincaré recurrences is related to the properties of the (right) eigenvectors $\psi(x, y)$ of the UPFO for the projected case. For this we determine the $x$-average of the eigenvector amplitude around a given position $x_{0}$ over a band of $1 \%$ width of the whole $x$-range: $\langle|\psi(y)|\rangle=100 M^{-1} \sum_{|\Delta x|<0.005} \mid \psi\left(x_{0}+\right.$ 

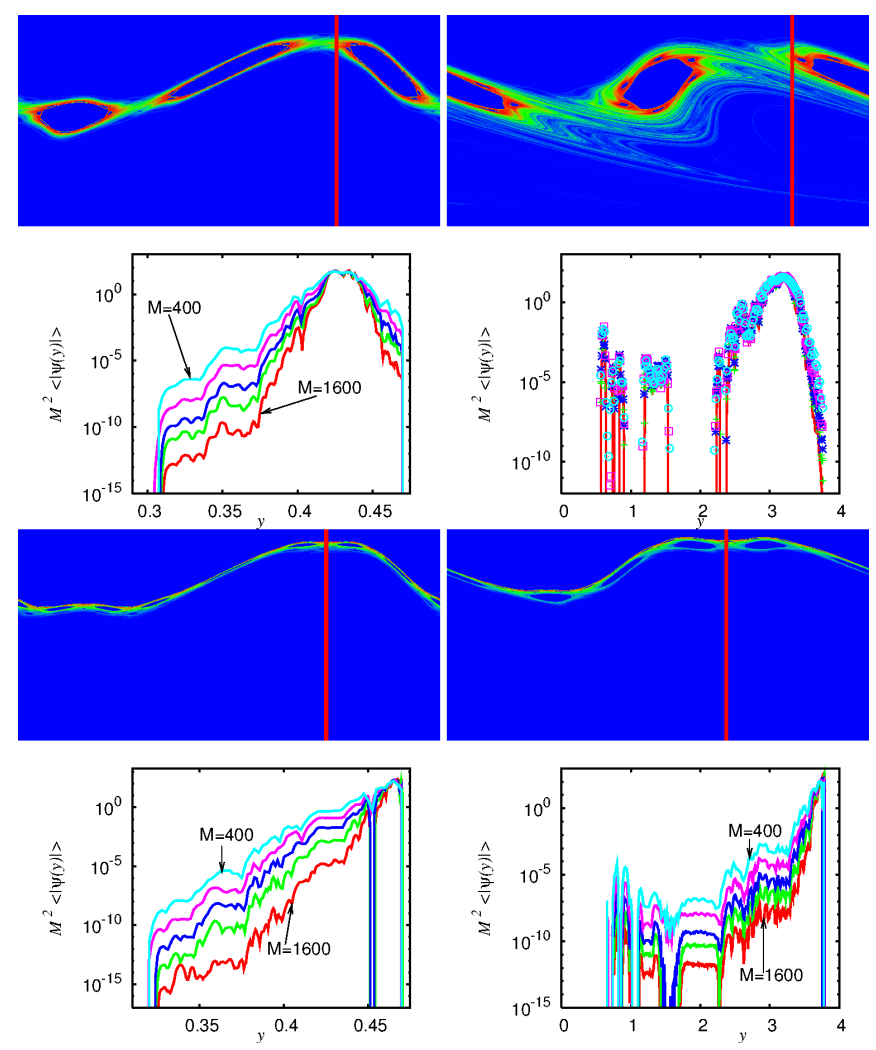

Fig. 14. (Color online) The localization properties in $y$ direction for certain eigenvectors of the UPFO for the projected case for the maps (1) (left column) and (3) (right column). The panels in the second and fourth row show the averaged modulus $\langle|\psi(y)|\rangle$ of the eigenvector components within a band of $1 \%$ width of the whole $x$-range at a certain $x=x_{0}$. The global structure of the corresponding eigenstates is shown in the corresponding first and third panels (counting from the top; the red vertical thick line indicates the range of $x$-values where the average has been performed for each $y$-value, $M=1600$ ). Data are shown for $M=400$ (cyan/highest curve), $M=560$ (pink/second curve), $M=800$ (blue/third curve), $M=1120$ (green/fourth curve) and $M=1600$ (red/lowest curve). In the right panel of the second row the data for different values of $M$ approximately coincide and only the data for $M=1600$ are shown by a full (red) curve; other $M$ values are shown as isolated data points for $M=1120$ (green crosses), $M=800$ (blue stars), $M=560$ (pink squares) and $M=400$ (cyan circles). For $M=1600$ the eigenvectors, shown in the density plots of the first and third row, correspond to the modes $\lambda_{4}$ and $\lambda_{31}$ of the map (1) (left column) and to the modes $\lambda_{2}$ and $\lambda_{17}$ of the map (3) (right column); for other $M$ we show corresponding eigenvector located at the same resonances.

$\Delta x, y) \mid$. The $y$-dependance of this average allows to visualize the localization properties of the eigenstate in $y$ direction. In Fig. 14 we show this quantity for two examples for each of the maps (1) and (3) and for different values of $M$ between 400 and 1600 .

For the case of the map (1), shown in the left column of Fig. 14, we see a clear evidence of exponential localization of eigenstates. In fact the average amplitude in a vicinity of $y \approx 0$, where the initial state is taken and where the absorption happens, has enormously small values being of the order of $10^{-15}$. These amplitudes on the tail drop significantly with an increase of $M$. For the map (3) the decay of eigenstates is more irregular since the band at $x \approx x_{0}$ crosses some secondary islands thus leading to appearance of a plateau in the decay with $y$. But in global we can still say that there is an exponential decay of eigenstates. This exponential localization of eigenstates reminds the Anderson localization in disordered solid state systems (see e.g. [49]).
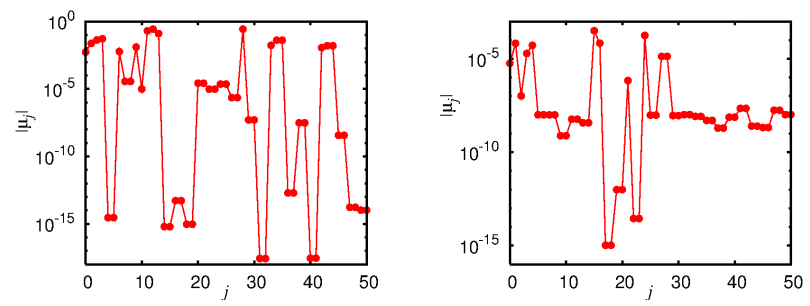

Fig. 15. (Color online) Modulus of the projection coefficients $\mu_{j}$ of the initial density vector $\psi_{\text {init }}$, localized in one cell at $x_{0}=y_{0}=0.0625$, with respect to the right eigenvectors $\psi_{j}^{R}$ (of the UPFO projected case for $M=1600$ ) versus level number $j$. These coefficients appear in the expansion $\psi_{\text {init }}=\sum_{j} \mu_{j} \psi_{j}^{R}$ (see text). The left and right panels represent data for the maps (1) and (3) respectively. The cases with $\left|\mu_{j}\right|=\left|\mu_{j+1}\right|$ correspond to pairs of complex conjugated modes with $\mu_{j+1}=\mu_{j}^{*}$.

We can also consider the projection of our initial state taken in a cell $\ell_{0}$ on the eigenstates. Indeed, this initial state can be expressed as $\psi_{\text {init }}=\sum_{j} \mu_{j} \psi_{j}^{R}$ where $\mu_{j}$ are expansion amplitudes and $\psi_{j}^{R}$ the right eigenvectors defined by Eq. (2). To determine the values of $\mu_{j}$ we need first to compute the left eigenvectors $\psi_{j}^{L}$ of the Ulam matrix $S_{p}$ which are biorthogonal to the right eigenvectors $\psi_{j}^{R}$ and provide the expansion amplitudes by the identity: $\left.\mu_{j}=<\psi_{j}^{L}\left|\psi_{\text {init }}>/<\psi_{j}^{L}\right| \psi_{j}^{R}\right\rangle$. Note that this expression does not depend on the choosen normalization of the eigenvectors and it requires only that $\left\langle\psi_{j}^{L} \mid \psi_{j}^{R}\right\rangle \neq 0$. However, for convenience, we have normalized both type of eigenvectors by the $L_{1}$-norm such that $\sum_{x, y}\left|\psi_{j}^{R, L}(x, y)\right|=$ 1 . We have numerically determined the first 51 left eigenvectors with the help of the Arnoldi method applied to the transpose of $S_{p}$ and therefore obtained the corresponding expansion amplitudes.

The dependence of $\mu_{j}$ on $j$ is shown in Fig. 15 . We see that there are enormously large fluctuations of $\mu_{j}$ which are in a range of 10 orders of magnitude. In particular the amplitudes corresponding to resonant modes are very small which is easy to understand if the resonant mode is localized far away from the initial state and does therefore not contribute to the expansion. We think that these fluctuations are at the origin of the slow algebraic decay of Poincaré recurrences $P(t)$ (see below).

In Fig. 16 we show the contribution of the largest $N_{m}$ eigenmodes to the statistics of Poincaré recurrences (for 

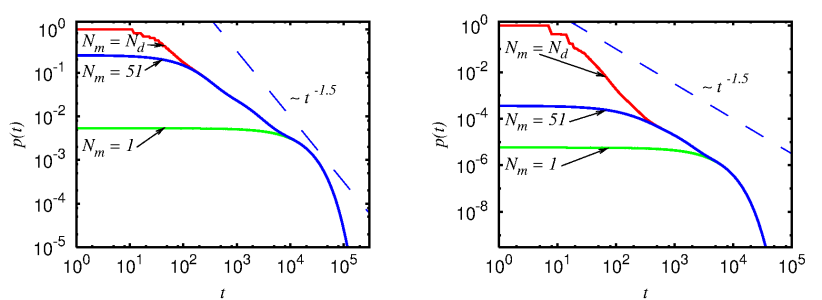

Fig. 16. (Color online) Contributions of the largest eigenmodes of the UPFO projected case at $M=1600$ to the statistics of Poincaré recurrences for the maps (1) (left panel) and (3) (right panel). Here, we show the probability $p(t)$ obtained from the expansion over eigenvectors given by the formula $p(t)=\sum_{j=0}^{N_{m}-1} p_{j} \lambda_{j}^{t}$ with $p_{j}=\mu_{j} \sum_{x, y} \psi_{j}^{R}(x, y), N_{m}$ being the number of used modes and the eigenvalues being ordered as $\left|\lambda_{0}\right|>\left|\lambda_{1}\right|>\left|\lambda_{2}\right|>\cdots$ (see text). The upper red curve is obtained from the direct iteration of the UPFO (see green curve in the right panels of figures 8 and 12 and corresponds to the contribution of the full spectrum of all eigenvalues with $N_{m}=N_{p}$. The middle blue curve corresponds to $N_{m}=51$ with the same $\mu_{j}$ values as those shown in figure 15 The main contributions to this curve arise from the diffusion modes (with real positive eigenvalues $\lambda_{j}>0$ ), the other resonant modes with complex or real negative eigenvalues give only a small contribution which does not modify the curve up to graphical precision. The bottom green curve corresponds to $N_{m}=1$, i. e. the contribution $\mu_{0} \lambda_{0}^{t}$ of the largest $\lambda$ eigenmode. In both panels the dashed line indicates for comparison a power law decay $P(t) \propto t^{-1.5}$

$M=1600)$ given by the formula: $p(t)=\sum_{j=0}^{N_{m}-1} p_{j} \lambda_{j}^{t}$ with $p_{j}=\mu_{j} \sum_{x, y} \psi_{j}^{R}(x, y)$ and the eigenvalues ordered as $\left|\lambda_{0}\right|>\left|\lambda_{1}\right|>\left|\lambda_{2}\right|>\cdots$.

For $N_{m}=N_{p}$, we have the statistics of Poincaré recurrences obtained from the iteration of the UPFO and already shown in Figs. 8 and 12 . For $N_{m}=51$ we have evaluated the sum using the expansion coefficients shown in Fig. 15. Both curves coincide at $t>10^{2}$ for the map (1) or at $t>3 \times 10^{2}$ for the map (3) showing that the largest eigenmodes determine the long time behavior. For large times $\left(t>10^{4}-10^{5}\right)$ only the first eigenmode contributes and the decay is purely exponential. It turns out that in the sum for $N_{m}=51$ the terms arising from the resonant modes can be omitted without changing the curve up to graphical precision since these modes contribute only very weakly in the expansion. In general, the partial sum $p(t)$ converges to the actual statistics of Poincaré recurrences $P(t)$ with increasing $N_{m}$ and at given value of $N_{m}$ one expects that $p(t)$ and $P(t)$ coincide for $t \gg 2 \gamma_{N_{m}}^{-1}$.

The data of Figs. 14, 15, 16 illustrate the nontrivial link between the localized eigenstates of the Ulam matrix and the decay of Poincaré recurrences. The eigenmodes are exponentially localized and for many of them their projection on the initial state is very small but at some large times their contribution can become very important since the modes with large projections decay more rapidly.

\section{Discussion}

Our studies show that the generalized Ulam method reproduces well the decay of Poincaré recurrences $P(t)$ in $2 \mathrm{D}$ symplectic maps with divided phase space. At the same time the computation of $P(t)$ is obtained in a more efficient way by the proposed SMCM allowing to reach time scales of the order of $t=10^{10}$. We find that at these large times the Poincaré exponent has values $\beta=1.58$ for the Chirikov standard map at $K_{g}$ and $\beta=1.70$ for the separatrix map at $\Lambda_{c}$. The recurrences at large times are dominated by sticking of trajectories not only in a vicinity of the critical golden curve but also in a vicinity of secondary resonance structures. This confirms earlier numerical observations obtained on shorter time scales [27.

The sticking around various different resonant structures on smaller and smaller scales of phase space leads to nontrivial oscillations of the Poincaré exponent. The values of $\beta$ found here are not so far from the average values found previously by averaging over maps at different parameters with $\beta \approx 1.5[18,19, \beta \approx 1.57$ [28. In agreement with the data presented here and in 34, we find that the above value of $\beta$ is close to the exponent of integrated density of states of the Ulam matrix which has $\beta \approx 1.5$. At the same time we see that at $t=10^{10}$ the fluctuations in the Chirikov standard map at various $N_{f}$ and various random realizations are significantly stronger as compared to the separatrix map.

We attribute these fluctuations to a localization of eigenstates of the Ulam matrix which gives very nontrivial properties of eigenstates projection on an initial state. The properties of these eigenstates are still poorly understood. We think that the further developments of analytical models of renormalization on Cayley type tree [22, 28, 32, 33 and their applications to the puzzle of statistics of Poincaré recurrences should develop a more detailed analysis of localization of eigenstates of the Ulam matrix.

This research is supported in part by the EC FET Open project "New tools and algorithms for directed network analysis" (NADINE No 288956). This work was granted access to the HPC resources of CALMIP (Toulouse) under the allocation 2012-P0110. We also acknowledge the France-Armenia collaboration grant CNRS/SCS No 24943 (IE-017) on "Classical and quantum chaos".

We dedicate this work to the memory of Boris Chirikov (06.06.1928 - 12.02.2008).

\section{References}

1. J. Loschmidt, Über den Zustand des Wärmegleichgewichts eines Systems von Körpern mit Röksicht auf die Schwerkraft, Sitzungsberichte der Akademie der Wissenschaften, Wien, Vol. II 73, 128 (1876)

2. L. Boltzmann, Über die Beziehung eines allgemeine mechanischen Satzes zum zweiten Haupsatze der Wärmetheorie, Sitzungsberichte der Akademie der Wissenschaften, Wien, Vol. II 75, 67 (1877)

3. V.I. Arnold, A. Avez, Ergodic problems of classical mechanics, Benjamin, Paris (1968). 
4. I.P. Cornfeld, S.V. Fomin, Y. G. Sinai, Ergodic theory, Springer, N.Y. (1982).

5. B.V. Chirikov, Research concerning the theory of nonlinear resonance and stochasticity, Preprint N 267, Institute of Nuclear Physics, Novosibirsk (1969) (in Russian) [Engl. Transl., CERN Trans. 71 - 40, Geneva, October (1971)].

6. B.V. Chirikov, Phys. Rep. 52, 263 (1979).

7. A.J.Lichtenberg, M.A.Lieberman, Regular and chaotic $d y-$ namics, Springer, Berlin (1992).

8. B.Chirikov, D.Shepelyansky, Scholarpedia, 3 (3), 3550 (2008)

9. J.M. Greene, J. Math. Phys. 20, 1183 (1979)

10. R.S. MacKay, Physica D 7, 283 (1983)

11. R.S. MacKay, I.C. Percival, Comm. Math. Phys. 94, 469 (1985)

12. B.V.Chirikov, Critical perturbation in standard map: a better approximation, arXiv:nlin/0006021 nlin.CD] (2000).

13. S.Aubry, Physica D 7, 240 (1983)

14. R.S.MacKay, J.D.Meiss, I.C.Percival, Physica D 13, 55 (1984)

15. J.M. Greene, R.S. MacKay, J.Stark, Physica D 21, 267 (1986)

16. H. Poincaré, Sur le problème des trois corps et les équations de la dynamique, Acta Mathematica 13, 1 - 270 (1890)

17. S.R. Chanon, J.L. Lebowitz, Ann. N.Y. Acad. Sci. 357, $108(1980)$

18. B. V. Chirikov, D. L. Shepelyansky, Preprint 81-69 Inst. Nuclear Physics, Novosibirk (1981) [English translation, Princeton Univ. Report No. PPPL-TRANS-133, (1983)]

19. Proc. IX Int. Conf. on Nonlinear Oscillations (Kiev 1981), Naukova Dumka 2, 420 (1984)

20. C. F. F. Karney, Physica D 8, 360 (1983)

21. B. V. Chirikov, D. L. Shepelyansky, Physica D 13, 395 (1984)

22. J. Meiss, E. Ott, Phys. Rev. Lett. 55, 2741 (1985); Physica D 20, 387 (1986)

23. B. V. Chirikov, D. L. Shepelyansky, in Renormalization Group Eds. D.V.Shirkov, D.I.Kazakov, A.A.Vladimirov, World Sci. Publ., Singapore p. 221 (1988)

24. B. V. Chirikov, D. L. Shepelyansky, Phys. Rev. Lett. 82 528 (1999)

25. B. V. Chirikov, D. L. Shepelyansky, Phys. Rev. Lett. 89, $239402(2002)$

26. M. Weiss, L. Hufnagel, R. Ketzmerick, Phys. Rev. Lett. 89, 239401 (2002)

27. M. Weiss, L. Hufnagel, R. Ketzmerick, Phys. Rev. E 67, 046209 (2003)

28. G. Cristadoro, R. Ketzmerick, Phys. Rev. Lett. 100, $184101(2008)$

29. R. Artuso, C. Manchein, Phys. Rev. E 80, 036210 (2009)

30. R. Venegeroles, Phys. Rev. Lett. Phys. Rev. Lett. 102, 064101 (2009)

31. I.I. Shevchenko, Phys. Rev. E 81, 066216 (2010)

32. V.A. Avetisov, S.K. Nechaev, Phys. Rev. E 81, 046211 (2010)

33. R. Ceder, O. Agam, Phys. Rev. E 87, 012918 (2013)

34. K.M. Frahm, D.L. Shepelyansky, Eur. Phys. J. B 76, 57 (2010)

35. K. Frahm, D.L. Shepelyansky (Eds.), Quantware Library, Section QNR16 at http://www.quantware.upstlse.fr/QWLIB/ulammethod/

36. S.M. Ulam, A Collection of mathematical problems, Vol. 8 of Interscience tracs in pure and applied mathematics, Interscience, New York, p. 73 (1960)
37. M. Brin, G. Stuck, Introduction to dynamical systems, Cambridge Univ. Press, Cambridge, UK (2002)

38. T.-Y. Li, J. Approx. Theory 17, 177 (1976)

39. Z. Kovács, T. Tél, Phys. Rev. A 40, 4641 (1989)

40. Z. Kaufmann, H. Lustfeld, J. Bene, Phys. Rev. E 53, 1416 (1996).

41. G. Froyland, R. Murray, D. Terhesiu, Phys. Rev. E 76, 036702 (2007)

42. G. Froyland, K. Padberg, Physica D 238, 1507 (2009)

43. L. Ermann, D.L. Shepelyansky, Physica D 241, 514 (2012)

44. D.L. Shepelyansky and O.V. Zhirov, Phys. Rev. E 81, 036213 (2010)

45. L.Ermann, D.L. Shepelyansky, Phys. Rev. E 81, 036221 (2010)

46. L.Ermann, D.L. Shepelyansky, Eur. Phys. J. B 75, 299 (2010)

47. D.L. Shepelyansky, Phys. Rev. E 77, 015202(R) (2008)

48. G. W. Stewart, Matrix Algorithms: Eigensystems, (SIAM, 2001), Vol. II

49. F. Evers and A. D. Mirlin, Rev. Mod. Phys. 80, 1355 (2008) 